\title{
Prevalence of Polycystic Ovarian Syndrome in Young Adult Unmarried Females Attending Zagazig University Hospital Outpatient Clinic
}

Soha Galal Siam, Badeea Seliem Soliman, Mohammed Ramadan Ali, Omnia Abdallah Mohamed Abdallah*

Department Obstetrics and Gynecology, Faculty of Medicine, Zagazig University, Sharkia, Egypt.

*Corresponding Author: Omnia Abdallah Mohamed Abdallah, Mobile: 01095967944, Email: abdallahomnia37@gmail.com

\begin{abstract}
Background: Polycystic ovary syndrome (PCOS) is one of the most prevalent endocrine and metabolic disorders in women of childbearing age.

Objective: To determine the frequency of Polycystic ovarian syndrome (PCOS) among young unmarried females to increase awareness of PCOS and to promote effective early medical interventions and healthy lives for women.

Methods: This was a prospective cross-sectional study in which 180 unmarried females between 16 to 24 years old attending gynecology and dermatology outpatient clinic suffering from menstrual abnormalities or symptoms of hyperandrogenism to estimate the prevalence rate of polycystic ovarian syndrome from May to November 2018. According to diagnostic criteria of the NIH, Rotterdam, and the AE-PCOS. Menstrual irregularities (MI) were identified, and clinical hyperandrogenism was evaluated by self-assessment of hirsutism using the modified Ferriman-Gallwey score. Blood analysis was done for measurement of prolactin, thyroid-stimulating hormone, and androgen hormones. Results: This study revealed Prevalence rate of PCOS is 55.6\% among all presented cases, oligomenorrhea, acne, and hirsutism were the most common complaint in this study. Also, this study showed that there is an association between PCOS and sedentary life, and unhealthy food habits. There was a significant difference between PCOS and non-PCOS in $\mathrm{LH}$ and prolactin levels. There is a disturbed LH $\backslash F S H$ ratio (2:1 or more). It also shows that there was a significant difference between PCOS and non-PCOS in ovarian volume.
\end{abstract}

Conclusions: Lifestyle modification as healthy food and exercise play important role in the prevention of PCOS.

Keywords: Polycystic Ovary Syndrome (PCOS), Hyperandrogenism (HA), Hirsutism, Menstrual Irregularities (MI).

\section{INTRODUCTION}

Polycystic ovarian syndrome (PCOS), also known as Stein-Leventhal syndrome, is a frequently occurring metabolic and reproductive endocrinopathy. Prevalence in the reproductive age group is reported in various studies as $5-20 \%{ }^{(1)}$. Young women with PCOS mainly present with reproductive problems such as hyperandrogenism, menstrual irregularities, infertility, and chronic anovulation. Infertility increases 10 folds in women with PCOS and affects up to $40 \%{ }^{(2)}$.

PCOS reduces fertility due to associated endocrine, metabolic and gynecological abnormalities that impact the quality and function of the ovary. Derangements in factors involved in normal follicular development lead to the arrest of follicular growth as the follicles reach a diameter of 4-8 $\mathrm{mm}$, the dominant follicle does not develop, and ovulation does not ensue. Increased insulin resistance which causes an increased LH/FSH ratio and decreased SHBG leads to hyperandrogenism. Studies have also reported poor pregnancy outcomes in these women ${ }^{(3)}$.

There is no single definitive test to diagnose PCOS, however, three different guidelines, National institute of health criteria (NIH), Androgen excessPCOS society of India criteria (AE-PCOS), or Rotterdam criteria can be followed ${ }^{(4)}$. Insulin resistance and obesity are considered intrinsic to PCOS but neither of them is included in the guidelines and should therefore be used for diagnostic purposes ${ }^{(5)}$.

Despite its high prevalence and implications on reproductive health, PCOS is underdiagnosed ${ }^{(6)}$.
PCOS is a heterogeneous disorder. There is a wide range in the age of manifestation and symptomatology. Recent trends of sedentary lifestyle, carbohydrate, and fat-rich foods predispose adolescent girls to weight gain and high BMI.

As a consequence, of these lifestyle changes, there is an increased risk of PCOS, including hormonal imbalance, menstrual problems, infertility, and altered pregnancy outcome in these women.

PCOS has a high prevalence in infertile women. Apart from its impact on reproductive outcome, PCOS also presents with delayed manifestations which have many potential metabolic and cardiovascular risks if not managed appropriately. Lifestyle modification at early age, counseling of the parents, better knowledge and attitude of women towards PCOS are crucial in improving the quality of life in women with PCOS ${ }^{(6)}$.

PCOS women present with a constellation of symptoms which significantly impacts the quality of life. These women are at higher risk for various morbidities such as obesity, insulin resistance, type II diabetes mellitus, cardiovascular disease (CVD), infertility, malignancy, and psychological disorders ${ }^{(7)}$.

This study aimed to determine the frequency of Polycystic ovarian syndrome (PCOS) among young unmarried females to increase awareness of PCOS and to promote effective early medical interventions and healthy lives for women.

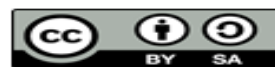

Received:19/6/2020

This article is an open access article distributed under the terms and conditions of the Creative Commons Attribution (CC BY-SA) license (http://creativecommons.org/licenses/by/4.0/) 


\section{PATIENTS AND METHODS}

Among 15300 cases attended Gynecology and Dermatology outpatient clinics at the time of the study from May to November 2018, there were 180 cases presented by symptoms suggesting polycystic ovarian syndrome $(1.17 \%)$. Only 100 cases were confirmed to be PCOS $(0.65 \%)$. These PCOS cases were classified according to age into two groups Group less than 20 years and another one more than 20 years. Diagnosis of (PCOS) below the age of 20 years depends on laboratory and clinical symptoms and signs of hyperandrogenism. While, Diagnosis of PCOS more than 20 years diagnosed by 2 of 3 Rotterdam criteria that include Clinical and laboratory symptoms and signs of hyperandrogenism, Ovulatory dysfunction, Polycystic ovaries on ultrasound.

\section{Inclusion criteria}

Age ranging from 16 to 24 years. Menstrual irregularity as oligomenorrhea, amenorrhea, and irregular cycles. Features of hyperandrogenism as hirsutism, acne, or androgenic alopecia. Obese girls (BMI of more than $30 \mathrm{~kg} / \mathrm{m}^{2}$ ).

\section{Exclusion criteria}

Participants with known malignancy such as virilizing tumors. Endocrinal disorders as Cushing syndrome, thyroid disorders, and adrenal disorders.

\section{Ethical Considerations:}

Written informed consent was obtained from all patients, the study was approved by the research ethics committee of the Faculty of Medicine, Zagazig University.

The work was carried out for studies involving humans following the World Medical Association's Code of Ethics (Helsinki Declaration).

A detailed history was taken from cases that included names, age, residence, and occupation. Menstrual history as the age of menarche, pattern, and frequency was taken. We also asked about exercise and eating habits (fast food, soft drinks, and chocolate eating).

The following clinical presentations were used to identify subjects with suspected PCOS: menstrual irregularities (MI), age of menarche, menstrual frequency, regularity, last menstrual period (LMP), and presence of dysmenorrhea. Menstrual abnormalities define chronic anovulation as amenorrhea of 3 months duration or oligomenorrhea (intermenstrual interval more than 35 days). Regular cycles were defined as 916 cycles of a 21-35 days duration within a year or no more than 4 days difference in duration between cycles (8).

Full clinical examinations were done (weight, height, waist circumference, BMI) also, vital signs (blood pressure, pulse, temperature, and respiratory rate).

All participants went through anthropometric examinations, including weight and height; body mass index (BMI) was calculated by dividing the weight $(\mathrm{kg})$ by the square meters of height (m2), and obesity was defined if BMI was $\geq 30 \mathrm{~kg} / \mathrm{m} 2$ according to WHO criteria.

Waist circumference by measurement was taken around the abdomen at the level of the umbilicus to measure waist circumference correctly. Body hair distribution to detect male pattern hair growth According to Ferriman Galloways.

Transabdominal ultrasound was done for cases more than 20 years, recent studies found that there is no role for ultrasound below the age of 20 and the diagnosis of PCOS below 20 years depend on clinical presentation (acne, hirsutism), ovulatory dysfunction, and the hormonal picture of hyperandrogenism as Testosterone and DHEA-S. Ultrasound was done between cycle day $2-7$ in menstruating females or after withdrawal bleeding in case of amenorrhea.

\section{Hormonal analysis}

A venous blood sample was taken by applying tourniquets on the patient arm to make the veins more prominent.

Basal hormonal detection, FSH, LH, Prolactin, DHEA-S, and Testosterone were done between cycle day 2-5 of menstruating females. In case of amenorrhea withdrawal, bleeding was done and a blood sample was taken 2-5 days of withdrawal bleeding. Withdrawal bleeding is done by oral progesterone pills (typically medroxyprogesterone, Provera,10mg oral daily for 10 days). After stopping the pills, the patient would be expected to have withdrawal bleeding.

Serum FSH, LH, and prolactin were done by Enzyme Immunoassay (EIA) kits. FSH: LH ratio was calculated. Serum FSH: LH 1:2 was taken as raised and below this was considered normal.

\section{Statistical Analysis}

Data collected throughout history, basic clinical examination, imaging, laboratory investigations, and outcome measures coded, entered, and analyzed using Microsoft Excel software. Data were then imported into Statistical Package for the Social Sciences (SPSS version 20.0). Software for analysis. According to the type of data, qualitative represent as number and percentage, quantitative represent by mean $\pm S D$, the following tests were used to test differences for significance difference and association of qualitative variable by Chi-square test $\left(\mathrm{X}^{2}\right)$. Differences between quantitative independent groups by t-test or Mann Whitney P value was set at $<0.05$ for significant results $\&<0.001$ for a highly significant result.

\section{RESULTS}

The most common gynecological complaint was oligomenorrhea and dermatological complain were acne and hirsutism. Figure 1

This study showed that the most common gynecological presentation in the PCOS group was 
oligomenorrhea while the most common dermatological presentations were acne and hirsutism. (Table 1)

This study showed that PCOS cases were significantly associated with fast food, soft drink, and chocolate eating (Table 2).

There was a statistically significant difference between the PCOS and non- PCOS women in LH and prolactin, but regarding testosterone, DHEA-S, and $\mathrm{FSH}$, there was no statistically significant difference (Table 3).

There were statistically significant differences between the PCOS and non- PCOS women below 20 years in $\mathrm{LH}$ and prolactin. But regarding testosterone,
DHEA-S, and FSH, there was no statistically significant difference. (Table 4)

There were statistically significant differences between the PCOS and non- PCOS females more than 20 years in $\mathrm{LH}$ and prolactin. But regarding testosterone, DHEA-S, and FSH, there were no statistically significant differences. (Table 5)

In this table, there was a statistically significant difference regarding follicle number 12 or more, peripheral distribution of follicles, and ovarian volume with no difference in highly echogenic bright ovarian stroma between PCOS and non PCOS women. (Table 6).

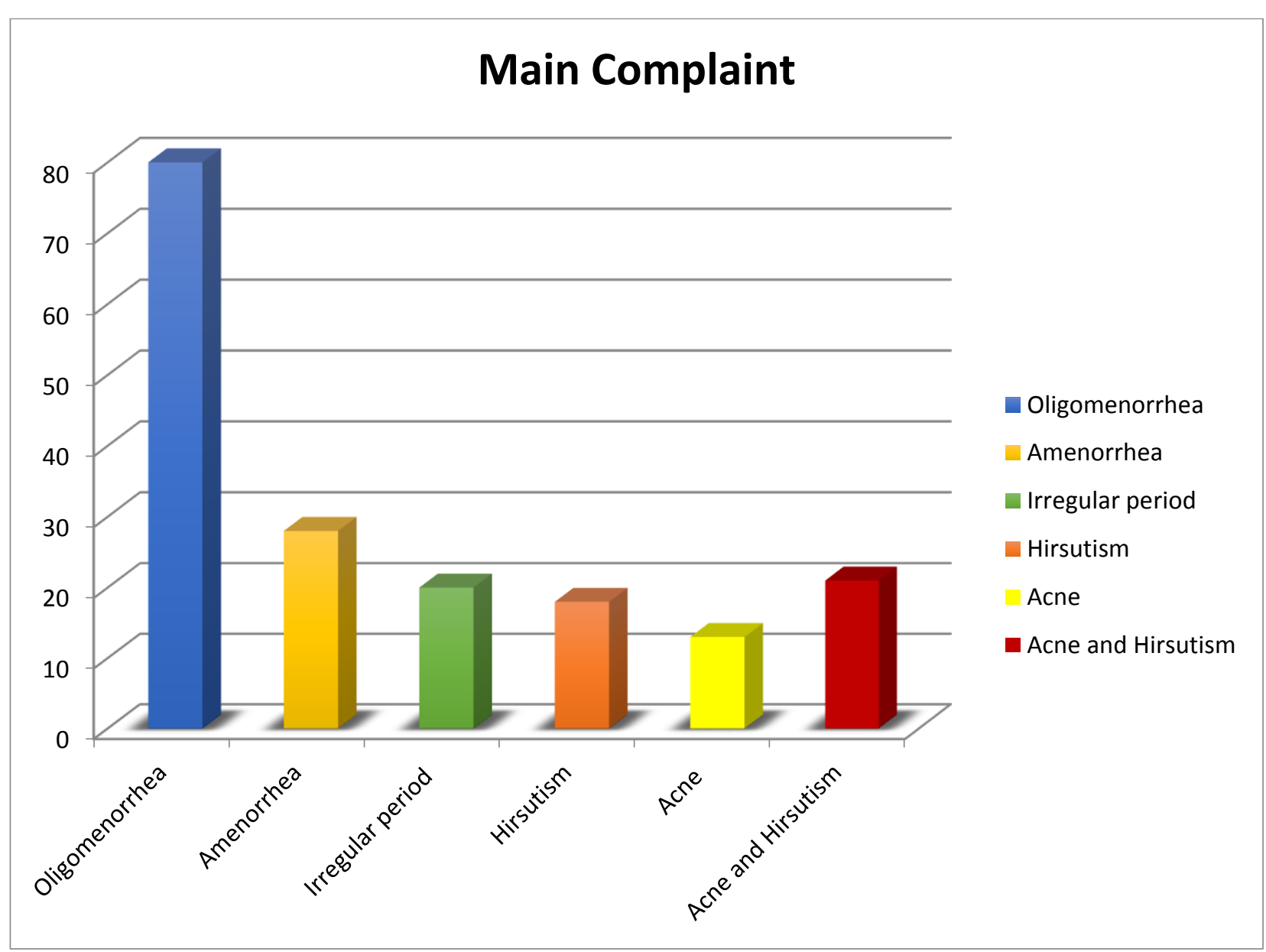

Figure 1: The main complaint among studied women

Table (1): Comparison of clinical presentation between groups

\begin{tabular}{|c|c|c|c|c|c|c|}
\hline Variable & \multicolumn{2}{|c|}{$\begin{array}{c}\text { PCOS }(\mathbf{1 0 0}) \\
\text { mean } \pm \text { SD }\end{array}$} & \multicolumn{2}{c|}{$\begin{array}{c}\text { non- PCOS (80) } \\
\text { mean } \pm \text { SD }\end{array}$} & $\mathbf{X}^{2}$ & P-value \\
\hline Complaint & $\mathbf{N}$ & $\boldsymbol{\%}$ & $\mathbf{N}$ & $\mathbf{0}$ & & \\
\hline Oligomenorrhea & $\mathbf{5 0}$ & $\mathbf{5 0 . 0 \%}$ & $\mathbf{3 0}$ & $\mathbf{3 7 . 5 \%}$ & $\mathbf{5 . 2 1}$ & $\mathbf{0 . 0 2}$ \\
Amenorrhea & $\mathbf{1 1}$ & $\mathbf{1 1 . 0 \%}$ & $\mathbf{1 7}$ & $\mathbf{2 1 . 2 5 \%}$ & $\mathbf{3 . 1 2}$ & $\mathbf{0 . 0 7 4}$ \\
Irregular period & $\mathbf{1 0}$ & $\mathbf{1 0 . 0 \%}$ & $\mathbf{1 0}$ & $\mathbf{1 2 . 5 \%}$ & $\mathbf{0 . 8 7}$ & $\mathbf{0 . 2 8}$ \\
Acne & $\mathbf{5}$ & $\mathbf{5 . 0 \%}$ & $\mathbf{8}$ & $\mathbf{1 0 . 0 \%}$ & $\mathbf{1 . 6 6}$ & $\mathbf{0 . 1 9}$ \\
Hirsutism & $\mathbf{1 1}$ & $\mathbf{1 1 . 0 \%}$ & $\mathbf{7}$ & $\mathbf{8 . 7 \%}$ & $\mathbf{0 . 7}$ & $\mathbf{0 . 4 1}$ \\
Acne and hirsutism & $\mathbf{1 3}$ & $\mathbf{1 3 . 0 \%}$ & $\mathbf{8}$ & $\mathbf{1 0 . 0 \%}$ & $\mathbf{0 . 3 9}$ & $\mathbf{0 . 5 3}$ \\
\hline
\end{tabular}


Table (2): Lifestyle habits comparison between studied groups

\begin{tabular}{|c|c|c|c|c|c|c|}
\hline \multirow{2}{*}{ Variable } & \multicolumn{2}{|c|}{ PCOS (100) } & \multicolumn{2}{|c|}{ non- PCOS (80) } & \multirow{2}{*}{$\mathrm{X}^{2}$} & \multirow{2}{*}{ P-value } \\
\hline & $\mathbf{N}$ & $\%$ & $\mathbf{N}$ & $\%$ & & \\
\hline $\begin{array}{c}\text { Daily sport } \\
\text { No } \\
\text { Yes }\end{array}$ & $\begin{array}{l}85 \\
15\end{array}$ & $\begin{array}{l}85 \% \\
15 \%\end{array}$ & $\begin{array}{l}60 \\
20\end{array}$ & $\begin{array}{l}75 \% \\
25 \%\end{array}$ & 2.87 & 0.09 \\
\hline $\begin{array}{c}\text { Healthy diet } \\
\text { No } \\
\text { Yes } \\
\end{array}$ & $\begin{array}{l}72 \\
28 \\
\end{array}$ & $\begin{array}{l}72 \% \\
28 \% \\
\end{array}$ & $\begin{array}{l}48 \\
32 \\
\end{array}$ & $\begin{array}{l}60 \% \\
40 \% \\
\end{array}$ & 2.88 & 0.08 \\
\hline $\begin{array}{c}\text { Fast food } \\
\text { No } \\
\text { Yes }\end{array}$ & $\begin{array}{l}46 \\
54\end{array}$ & $\begin{array}{l}46 \% \\
54 \%\end{array}$ & $\begin{array}{l}59 \\
21 \\
\end{array}$ & $\begin{array}{l}73.7 \% \\
26.3 \% \\
\end{array}$ & 14.08 & $0.0001 * *$ \\
\hline $\begin{array}{c}\text { Soft drink } \\
\text { No } \\
\text { Yes } \\
\end{array}$ & $\begin{array}{l}22 \\
78 \\
\end{array}$ & $\begin{array}{l}22 \% \\
78 \% \\
\end{array}$ & $\begin{array}{l}50 \\
\mathbf{3 0} \\
\end{array}$ & $\begin{array}{l}62.5 \% \\
37.5 \% \\
\end{array}$ & 30.3 & $\mathbf{0 . 0 0} * *$ \\
\hline $\begin{array}{c}\text { Chocolate eating } \\
\text { No } \\
\text { Yes }\end{array}$ & $\begin{array}{l}55 \\
45\end{array}$ & $\begin{array}{l}55 \% \\
45 \%\end{array}$ & $\begin{array}{l}66 \\
14 \\
\end{array}$ & $\begin{array}{l}82.5 \% \\
17.5 \%\end{array}$ & 15.22 & $0.00 * *$ \\
\hline
\end{tabular}

Table (3): Hormonal levels comparison between PCOS and non- PCOS females:

\begin{tabular}{|l|c|c|c|c|}
\hline \multicolumn{1}{|c|}{ Variable } & $\begin{array}{c}\text { PCOS }(100) \\
\text { mean } \pm \text { SD }\end{array}$ & $\begin{array}{c}\text { non- PCOS (80) } \\
\text { mean } \pm \text { SD }\end{array}$ & $\begin{array}{c}\text { test } \\
\text { M.W }\end{array}$ & P-value \\
\hline FSH(mIU/L): & $6.2 \pm 1.1$ & $5.5 \pm 1.4$ & 1.6 & 0.089 \\
\hline LH(mIU/L) & $13.5 \pm 2.4$ & $9.1 \pm 1.6$ & 4.8 & $0.001 * *$ \\
\hline Testosterone (ngm/l) & $2.05 \pm 0.8$ & $1.3 \pm 0.1$ & 1.3 & 0.1 \\
\hline Prolactin level (ngm/l) & $17.3 \pm 3.8$ & $10.1 \pm 2.6$ & 7.6 & $0.001 * *$ \\
\hline DHEA-S (ngm/l) & $6.2 \pm 1.8$ & $5.1 \pm 1.4$ & 1.5 & 0.13 \\
\hline
\end{tabular}

M.W=Mann-Witenny U test. $\quad * *$ Statistically highly significant difference $(\mathrm{P} \leq 0.001)$

Table (4): Hormonal level comparison between PCOS and non- PCOS women less than 20 years

\begin{tabular}{|c|c|c|c|c|}
\hline Variable & $\begin{array}{c}\text { PCOS }(60) \\
\text { mean } \pm \text { SD }\end{array}$ & $\begin{array}{c}\text { non- PCOS (50) } \\
\text { mean } \pm \text { SD }\end{array}$ & $\begin{array}{c}\text { test } \\
\text { M.W }\end{array}$ & P-value \\
\hline FSH(mIU/L): & $6.1 \pm 1.8$ & $5.7 \pm 1.8$ & 1.5 & 0.082 \\
\hline LH(mIU/L) & $13.7 \pm 2.2$ & $8.8 \pm 2.6$ & 5.1 & $0.001^{* *}$ \\
\hline Testosterone (ngm/l) & $2.03 \pm 0.12$ & $1.5 \pm 0.08$ & 1.6 & 0.099 \\
\hline Prolactin & $17.1 \pm 3.4$ & $9.8 \pm 1.6$ & 8.2 & $0.0003 * *$ \\
\hline DHEA-S (ngm/l) & $5.1 \pm 0.3$ & $4.7 \pm 1.1$ & 1.1 & 0.24 \\
\hline
\end{tabular}

Table (5): Hormonal level comparison between PCOS and non- PCOS females for more than 20 years:

\begin{tabular}{|c|c|c|c|c|}
\hline Variable & $\begin{array}{c}\text { PCOS (40) } \\
\text { mean } \pm \text { SD }\end{array}$ & $\begin{array}{c}\text { non- PCOS (30) } \\
\text { mean } \pm \text { SD }\end{array}$ & $\begin{array}{c}\text { test } \\
\text { M.W }\end{array}$ & P-value \\
\hline FSH (mIU/L): & $6.4 \pm 1.5$ & $5.8 \pm 1.1$ & 1.51 & 0.096 \\
\hline LH (mIU/L) & $13.7 \pm 3.7$ & $9.3 \pm 2.8$ & 4.3 & $0.002^{*}$ \\
\hline $\begin{array}{c}\text { Testosterone } \\
(\text { ngm/l) }\end{array}$ & $2.14 \pm 0.2$ & $1.5 \pm 0.12$ & 1.6 & 0.089 \\
\hline $\begin{array}{c}\text { Prolactin } \\
\text { level(ngm/l) }\end{array}$ & $17.8 \pm 4.2$ & $11.2 \pm 3.2$ & 6.2 & $0.001 * *$ \\
\hline DHEA-S (ngm/l) & $6.3 \pm 1.9$ & $5.3 \pm 1.7$ & 1.6 & 0.12 \\
\hline
\end{tabular}

$* *$ Statistically highly significant difference $(\mathrm{P} \leq 0.001)$ 
Table (6): Hormonal level comparison between PCOS and non- PCOS females for more than 20 years:

\begin{tabular}{|l|c|c|c|c|c|c|}
\hline \multicolumn{1}{|c|}{ Variable } & $\begin{array}{c}\text { PCOS } \\
\text { NO (40) }\end{array}$ & $\%$ & $\begin{array}{c}\text { Non- } \\
\text { PCOS NO } \\
(30)\end{array}$ & $\%$ & X2 & P-value \\
\hline Follicle number>12 (2-9mm) & 34 & $\mathbf{8 5 . 0 \%}$ & $\mathbf{1 0}$ & $\mathbf{3 3 . 3 \%}$ & $\mathbf{2 2 . 1}$ & $\mathbf{0 . 0 0} * *$ \\
\hline Peripheral distribution of follicles & 30 & $\mathbf{7 5 . 0 \%}$ & $\mathbf{8}$ & $\mathbf{2 6 . 7 \%}$ & $\mathbf{2 3 . 7}$ & $\mathbf{0 . 0 0} * *$ \\
\hline Ovarian volume of more than 9cm3 & $\mathbf{3 0}$ & $\mathbf{7 5 . 0 \%}$ & $\mathbf{7}$ & $\mathbf{2 3 . 3 \%}$ & $\mathbf{2 7 . 5}$ & $\mathbf{0 . 0 0} * *$ \\
\hline $\begin{array}{l}\text { Highly echogenic bright ovarian } \\
\text { stroma }\end{array}$ & 25 & $\mathbf{6 2 . 5 \%}$ & $\mathbf{1 2}$ & $\mathbf{4 0 . 0 \%}$ & $\mathbf{4 . 8}$ & $\mathbf{0 . 0 2 *}$ \\
\hline
\end{tabular}

$* *$ Statistically highly significant difference $(\mathrm{P} \leq 0.001)$

\section{DISCUSSION}

In our study among 15300 cases attended Gynecology and dermatology outpatient clinics at the time of the study, there were 180 cases presented by symptoms suggesting PCOS (1.17\%), only 100 cases were confirmed to be PCOS $(0.65 \%)$ as they fulfilled the postulated criteria for PCOS diagnosis. Prevalence of PCOS defined on basis of Rotterdam criteria was therefore estimated at (55\%) among the presented cases (180). This is much higher than prevalence rates reported by a recent meta-analysis which concluded that the overall prevalence of PCOS (with corresponding $95 \% \mathrm{CI}$ ) according to diagnostic criteria of the NIH, Rotterdam and the AE-PCOS Society is $6 \%(5-8 \%, \mathrm{n}=18$ trials $), 10 \%(8-13 \%, \mathrm{n}=15$ trials $)$ and $10 \%(7-13 \%, \mathrm{n}=10$ trials), respectively(9). Indeed, prevalence rates were reported in the literature up to $20 \%$ in adults, and up to $30 \%$ in adolescent females ${ }^{(10)}$.

The hallmarks of the clinical presentations of PCOS include menstrual irregularity, hyperandrogenism, and cystic ovaries. Nevertheless, the pattern of clinical presentation varies greatly among the affected women ${ }^{(11)}$. The current study indicated that menstrual irregularities including oligomenorrhea and amenorrhea were also the most prevalent clinical presentation in the study population (44.4\%, 15.5\%; respectively). Analyzing PCOS group only, menstrual disorders were the most prevalent clinical presentation $(71 \%)$, while hyperandrogenism was identified in $29 \%$ of the cases. Similar to our findings, Milczarek et al. ${ }^{(12)}$ evaluated 26 girls with menstrual disorders and/or symptoms of hyperandrogenism two years after menarche and applied Rotterdam criteria for possible diagnosis of PCOS. The study group was divided into three subgroups: with isolated cutaneous manifestation $(\mathrm{CM})$ of hyperandrogenism $(\mathrm{n}=6)$, with isolated menstrual disorders $(\mathrm{n}=10)$, and with cutaneous manifestation and menstrual disorders $(\mathrm{n}=10)$. PCOS cases presented with the highest proportion in the menstrual disorders group (70\%). By more restricted criteria with a testosterone cut-off point of $>55 \mathrm{ng} / \mathrm{dl}$, only seven patients (27\%) met all Rotterdam criteria.

Similarly, another study was done by VelijaAšimi, Zelija ${ }^{(13)}$ to evaluate the endocrine changes in PCOS women during metformin treatment which was done on 100 women with PCOS aged 20-40 years, this study reported oligomenorrhea or amenorrhea in $69 \%$ of their study population.

On contrary, Thathapudi et al. ${ }^{(14)}$ reported higher rates of menstrual disorders (89\%) in the PCOS study group. This discrepancy could be interpreted on basis of the strict inclusion criteria of their study population, and the selection of the PCOS patients attending only the infertility clinics. Furthermore, a higher prevalence of menstrual irregularities was also reported by Yetim et al. ${ }^{\left({ }^{10)}\right.}$ who identified $83 \%$ of the subjects with PCOS were oligomenorrheic/amenorrheic. Comparing PCOS to the non-PCOS group, the current results indicated a statistically significant difference in percentages of oligomenorrhea $(p=0.02)$. Nevertheless, other clinical presentations including amenorrhea, irregular periods, acne, and hirsutism were similar to control ( $p>0.05$ ). The difference in reported rates of PCOS clinical presentations may differ among the studies for many reasons, principally due to differences in the anthropometrics and hormonal profiles of the included females ${ }^{(15)}$.

Interestingly, lifestyle pattern demonstrated a significant relationship with PCOS. The current analysis indicated significantly higher consumption of fast food $(\mathrm{P}<0.0001)$ in PCOS compared to non-PCOS (54\% vs $26.3 \%$; respectively).

Nevertheless, comparing unhealthy diet patterns in both groups did not show a statistical significance $(\mathrm{p}=0.08)$. This finding should be interpreted with caution; as it was reported that women with PCOS may have a greater appetite and are more overweight, despite a healthier diet ${ }^{(\mathbf{1 6})}$.

A recent meta-analysis investigated the effect of lifestyle on reproductive, anthropometric (weight and body composition), metabolic, and quality of life factors in PCOS included 15 randomized controlled trials (RCTs) comparing lifestyle treatment (diet, exercise, behavioral or combined treatments) to minimal or no treatment in women with PCOS in a total of 498 participants. The main finding was that lifestyle intervention may significantly improve the free androgen index (FAI), weight, and BMI in women with PCOS ${ }^{(17)}$. Association between major dietary patterns and polycystic ovary syndrome was investigated by Shahdadian $\boldsymbol{e t}$ al. ${ }^{(18)}$, who reported 
that Western and plant-based dietary patterns were associated with an increased risk of PCOS. Also, moderate adherence to the mixed dietary pattern was associated with a reduced risk of PCOS. Similarly, a cross-sectional cohort study of 711 women with PCOS from a heterogeneous population spanning a wide spectrum of socioeconomic strata. There was a notable association between increased calories and fat content in the diet with the presence of hirsutism, raised body mass index, insulin resistance ${ }^{(19)}$.

Chocolate consumption in the study carried by Graff et $\boldsymbol{a l}$. $^{(20)}$, was significantly higher in the PCOS group $(45 \%$ vs $17.5 \%, \mathrm{p}<0.001)$. The possible association between chocolate intake and PCOS may be emphasized on basis of the high glycemic index contained within the chocolate, resulting in increased BMI and worsening of insulin resistance. Indeed, the effects of chocolate intake were little investigated in the PCOS context. However, reports demonstrated conflicting evidence concerning its impact on PCOS patients. Dark chocolate is rich in cocoa which is a rich source of dietary polyphenols. Polyphenols are known for their antioxidant properties and were associated with improved insulin resistance ${ }^{(21)}$. Also, it was shown that acute dark chocolate consumption before prolonged exercise enhanced insulin sensitivity compared with chocolate consumption alone ${ }^{(22)}$. The precise impact of chocolate consumption on PCOS is therefore still questionable.

Physical activity may also play a role in PCOS pathology. The current findings indicate that lower application of daily exercise in PCOS group vs nonPCOS group ( $15 \%$ vs $25 \%$; $\mathrm{p}=0.08$ ). Despite statistical non-significance, these results shouldn't be overlooked. In line with the current results, a recent study carried by Bialka-Kosiec et al. ${ }^{(23)}$ in which they examined 37 participants with a diagnosis of PCOS, and 48 healthy control females with no prior diagnosis of PCOS concluded that the young female participants with PCOS were shown to have similar body composition to age-matched healthy controls. However, the clinical group with PCOS reported less frequent use of exercise. Statistical non-significance depicted in our work doesn't preclude the role of physical activity, instead, they may be related to the lower percentages identified throughout our cohort with regular exercise attributed to the different definitions of "exercise" component in relevant studies. For example, Bialka-Kosiec et al. ${ }^{(23)}$ assigned subjects to have exercise or not based on a subjective questionnaire, on contrary, our work addressed only females with regular daily aerobic exercise. The strict criteria for exercise pattern in the current study were associated with lower sample size and a possible increase in type II statistical errors with subsequent statistical non-significance. Samadi et al. ${ }^{(24)}$, recruited 30 patients with PCOS having age between 20 and 35 and body mass index $(\mathrm{BMI}) \geq 30 \mathrm{~kg} / \mathrm{m} 2$ selected based on Rotterdam Diagnostic Criteria, and randomly divided into experimental (high-intensity exercise + metformin, $\mathrm{N}=15$ ) and control groups (metformin, $\mathrm{N}$ $=15)$.

The exercises were done for 12 weeks. After 12 weeks, no significant difference was observed in waistto-hip ratio (WHR), but in the exercise group, the BMI and fat mass significantly decreased and levels of follicle-stimulating hormone (FSH), free testosterone (FT) increased compared to the control group ( $\mathrm{P}<$ 0.05). While levels of improvement of total testosterone (TT), dehydroepiandrosterone sulfate (DHEAS), and luteinizing hormone (LH) were not significant between the two groups $(\mathrm{P}>0.05)$. Furthermore, there was a significant decrease in the homeostatic model assessment of insulin resistance (HOMA-IR) and hirsutism severity in the experimental group $(\mathrm{P}<0.05)$. In both groups, the order of menstrual cycles improved significantly $(\mathrm{P}<0.05)$. These findings suggest that even when significant differences exist, the effects of exercise may not be homogenous across the whole components of PCOS, raising concerns about the real value of exercise in improving the syndrome.

In this study, analysis of the difference in hormonal profiles comparing PCOS subjects to nonPCOS ones demonstrated significant differences in mean LH levels (13.5 vs 9.1 IU; respectively) and prolactin levels (17.3vs $10.1 \mathrm{ng} / \mathrm{ml}$; respectively). FSH, Testosterone levels and DHEA-S failed to demonstrated significance upon comparison $(\mathrm{p}=0.089$, $0.1,0.13$; respectively). This pattern was maintained on subgroup analysis for females more than or less 20 years confirming independence of hormonal changes on age. Similarly, Yue et al. ${ }^{(25)}$, reported a nonsignificant difference in FSH levels comparing PCOS to healthy controls in line with the current findings. In a similar context, Dambala et al. ${ }^{(26)}$ analyzed the results of 42 women with PCOS and 42 controls, matched for age and weight. Comparing PCOS to control, they found a non-significant difference in FSH, DHEA-S, and total testosterone levels.

Different results were reported by Yetim $\boldsymbol{e t}$ al. (10), who analyzed the results of hormonal profiles of 53 adolescent girls aged between 14.5 and 20 years who were admitted to their outpatient clinic with symptoms of hirsutism and/or irregular menses and diagnosed as having PCOS following the Rotterdam criteria. The LH levels and LH/FSH ratio were significantly higher ( $\mathrm{p}=0.005, \mathrm{p}=0.042$; respectively) and $\mathrm{SHBG}$ was significantly lower $(\mathrm{p}=0.004)$ in PCOS patients as compared to the controls.

Strikingly, the significantly higher levels of prolactin presented in the PCOS cohort in the current results may be only attributed to false diagnosis PCOS due to symptomatic similarities between PCOS and hyperprolactinemia. Recent studies that have conducted a rigorous etiological investigation show that hyperprolactinemia found in PCOS correspond either to a non-permanent increase of prolactin levels, 
to prolactin-secreting pituitary adenoma, or other etiologies ${ }^{(27)}$. For instance, Hayashida et al. ${ }^{(28)}$, studied a population of 227 PCOS women. $6 \%$ of PCOS women had elevated prolactin $(n=16)$, which was consistently explained by the presence of pituitary adenoma.

Assessment of biochemical markers of hyperandrogenemia through the current work resulted in non-significant differences comparing PCOS hormonal profiles with non-PCOS ones as regarding testosterone levels and DHEA-S ( $\mathrm{p}=0.1, \quad 0.24$; respectively) Similar results were reported by Milczarek et al. ${ }^{(12)}$ in which they carried subgroup analysis in different ages has replicated the same pattern of non-significance in subjects more or less than 20 years. Strikingly, other reports indicated that PCOS has significantly higher testosterone and DHEA-S ${ }^{(14,25)}$.

The ultrasonographic features of adult PCOS were well established through the current work. Assessment of ovarian morphology in 40 adult PCOS cases based on US findings in the current study resulted in significant differences between PCOS and non-PCOS groups. Ovarian volumes (OV) were statistically higher in PCOS $(p=0.0003)$ with the majority of cases $(85 \%)$ presented with 12 or more follicles measuring 2-9 $\mathrm{mm} 3$, and ovarian volume of more than $9 \mathrm{~cm} 3$ with peripheral distribution was presented in $30(75 \%)$ patients. In accordance with our findings, Sujata et al. ${ }^{(29)}$, evaluated 26 PCOS women (Rotterdam criteria) and 45 matched ovulatory and normo-androgenic women. A detailed 2D and 3D scan was carried out in the early follicular phase (Day 2-Day 5 ) in all patients. Ovarian volume, follicle number per ovary (FNPO), and stromal volume were measured in PCOS and controls. Mean ovarian volume was $13.7 \pm 5.89$ and $5.06 \pm 2.44(\mathrm{p}<0.0001)$, FNPO was $19.18 \pm 6.89$ and $7.13 \pm 3.51(\mathrm{p}<0.0001)$ in PCOS and controls, respectively. The cut-offs for the diagnosis of PCOS were $2 \mathrm{D} \mathrm{OV}=6.15 \mathrm{~cm} 3,2 \mathrm{D} F \mathrm{FPO}=12$. By 3D scan, $\mathrm{OV}=7 \mathrm{~cm} 3, \mathrm{FNPO}=10$, stromal volume $=6 \mathrm{~cm} 3$. Importantly, they reported that 2D FNPO showed the highest specificity and sensitivity (AUC), 0.95238 , and 0.93548 , for the diagnostic accuracy of PCOS. Furthermore, Şentürk et al. ${ }^{(30)}$, conducted a prospective randomized control study of 40 PCOS patients and 40 healthy women who were matched concerning BMI $\left(<25 \mathrm{~kg} / \mathrm{m}^{2}\right.$ and $\left.\geq 25 \mathrm{~kg} / \mathrm{m}^{2}\right)$. When compared to healthy women, PCOS patients had significantly higher ovarian volumes $(\mathrm{P}=0.01)$. Interestingly, pelvic MRI exams of obese adolescent girls whose examination is difficult using pelvic US techniques showed also increased ovarian volumes and stroma for PCOS cases ${ }^{(31)}$. In a similar context, a study carried by Aydogmus et al. ${ }^{(32)}$ in which they reported that average follicle number per ovary presented statistical significance difference comparing PCOS to healthy controls $(\mathrm{P}<0.001)$, and was found to be significantly correlated with $\mathrm{AMH}$, as a sensitive marker of PCOS diagnosis. It should be noted that studies similar to our work yielded different mean follicle counts per ovary in PCOS cases. This can be interpreted considering that the latest generation of ultrasound devices is more sensitive and yields a larger follicle count in the general population; therefore, the current use of the old cutoff can overestimate the prevalence of PCOM. And so, a group of experts proposed the use of higher thresholds (that is, 19 to 25 follicles per ovary) to define PCOM with the new ultrasound machines ${ }^{(33)}$.

\section{CONCLUSIONS}

Polycystic ovarian syndrome is a common problem of women of reproductive age affecting their life physically mentally and socially. Lifestyle modification as healthy food and exercise play important role in the prevention of PCOS.

Future studies should be designed to include patients from different centers using more sensitive assays or more recent markers to externally validate the study results.

\section{REFERENCES}

1. Melo A, Ferriani R, Navarro $P$ (2015): Treatment of infertility in women with polycystic ovary syndrome: approach to clinical practice. Clinic., 70(11):765-9.

2. Hart R, Doherty D (2015): The potential implications of a PCOS diagnosis on a woman's long-term health using data linkage. J Clinic Endocrinol Metabol., 100(3):911-9.

3. Kamangar F, Okhovat J, Schmidt T et al. (2015): Polycystic ovary syndrome: special diagnostic and therapeutic considerations for children. Pediatr Dermatol., 32(5):571-8.

4. Azziz R, Carmina E, Dewailly D et al. (2009): The Androgen Excess and PCOS Society criteria for the polycystic ovary syndrome: the complete task force report. Fertil Steril., 91(2):456-88.

5. Witchel S, Oberfield S, Rosenfield $R$ et al. (2015): The diagnosis of polycystic ovary syndrome during adolescence. Hormone Res Paediatr., 83(6):376-89.

6. Patil S, Ramesh S, Murthy Kharidhi H (2019): A prospective analysis of polycystic ovarian syndrome in infertile women. Int. J. Reprod. Contracept Obstetric Gynecol., 2320-1770.

7. El Hayek S, Bitar L, Hamdar L et al. (2016): Polycystic ovarian syndrome: an updated overview. Front Physiol., 7:124-8.

8. Elting $\mathrm{M}$, Korsen $\mathrm{T}$, Rekers-Mombarg $\mathrm{L}$ et al. (2000): Women with polycystic ovary syndrome gain regular menstrual cycles when aging. Hum Reprod., 15(1), 24-28.

9. Bozdag G, Mumusoglu S, Zengin D et al. (2016): The prevalence and phenotypic features of polycystic ovary syndrome: a systematic review and meta-analysis. Hum Reprod., 31(12): 2841-55.

10. Yetim A, Yetim Ç, Baş F et al. (2016): Anti-müllerian hormone and inhibin-a, but not inhibin-b or insulin-like peptide-3, may be used as surrogates in the diagnosis of polycystic ovary syndrome in adolescents: preliminary results. J Clin Res Pediatr Endocrinol., 8(3): 288-93.

11. Ndefo U, Eaton A, Green M et al. (2013): Polycystic 
ovary syndrome: a review of treatment options with a focus on pharmacological approaches. Pharmacy and Therapeutics, 38(6): 336-43.

12. Milczarek M, Kucharska A, Borowiec A (2019): Difficulties in diagnostics of polycystic ovary syndrome in adolescents--a preliminary study. Pediatr Endocrinology, Diabetes \& Metabolism, 25(3): 122-26.

13. Velija-Ašimi $Z$ (2013): Evaluation of endocrine changes in women with the polycystic ovary syndrome during metformin treatment. Bosn J Basic Med Sci., 13(3):180-85.

14. Thathapudi S, Kodati V, Erukkambattu J et al. (2014): Anthropometric and biochemical characteristics of the polycystic ovarian syndrome in South Indian women using AES-2006 criteria. Int J Endocrinol Metab., 12(1): 16-22.

15. Hsu M (2015): Clinical characteristics in Taiwanese women with polycystic ovary syndrome. Clin Exp Reprod Med., 42(3): 86-94.

16. Moran L, Grieger J, Mishra G et al. (2015): The association of a Mediterranean-style diet pattern with polycystic ovary syndrome status in a community cohort study. Nutrients, 7(10): 8553-64.

17. Lim S, Hutchison S, Van Ryswyk E et al. (2019): Lifestyle changes in women with polycystic ovary syndrome. https://pubmed.ncbi.nlm.nih.gov/30921477/

18. Shahdadian $F$, Ghiasvand $R$, Abbasi $B$ et al. (2019): Association between major dietary patterns and polycystic ovary syndrome: evidence from a casecontrol study. Appl Physiol Nutr Metab., 44(1), 52-8.

19. Kulkarni S, Patil A, Gudi A et al. (2019): Changes in diet composition with urbanization and its effect on the polycystic ovarian syndrome phenotype in a Western Indian population. Fertil Steril., 112(4), 758-63.

20. Graff S, Mário F, Alves B et al. (2013): Dietary glycemic index is associated with less favorable anthropometric and metabolic profiles in polycystic ovary syndrome women with different phenotypes. Fertili Sterili., 100(4), 1081-8.

21. 21-Andujar I, Recio M, Ginor R et al. (2012): Cocoa polyphenols and their potential benefits for human health. Oxid Med Cell Longev., 2012:5-7.

22. Davison G, Callister R, Williamson G et al. (2012): The effect of acute pre-exercise dark chocolate consumption on plasma antioxidant status. Eur J Nutr., 51: 69-79.

23. Bialka-Kosiec Agnieszka A, Krzysztof W, Magdalena P et al. (2019): Body Mass Composition and Dietary Habits in Adolescents with Polycystic Ovary Syndrome. Ginekologia Polska , 90 (10): 58595.
24. Bialka-Kosiec A, Wilk K, Pytel M et al. (2019): Body mass composition and dietary habits in adolescents with polycystic ovary syndrome. Ginekol Pol., 90(10), 58995.

25. Samadi Z, Bambaeichi E, Valiani M et al. (2019): Evaluation of changes in levels of hyperandrogenism, hirsutism and menstrual regulation after a period of aquatic high-intensity interval training in women with polycystic ovary syndrome. Int J Prev Med., 10: 187192.

26. Yue C, Lu L, Li M et al. (2018): Threshold value of anti-Mullerian hormone for the diagnosis of polycystic ovary syndrome in Chinese women. PLoS One, 13(8): e0203129.

27. Dambala K, Vavilis D, Bili E et al. (2017): Serum visfatin, vascular endothelial growth factor, and matrix metalloproteinase-9 in women with polycystic ovary syndrome. Gynecol Endocrinol., 33(7): 529-33.

28. Delcour C, Robin G, Young J et al. (2019): PCOS and Hyperprolactinemia: what do we know in 2019?. Clin Med Insights Reprod Health, 13: 1179-83.

29. Hayashida S, Marcondes J, Soares J et al. (2014): Evaluation of macroprolactinemia in 259 women under investigation for polycystic ovary syndrome. Clin Endocrinol., 80(4): 616-8.

30. Sujata K, Swoyam S (2018): $2 D$ and $3 D$ transvaginal sonography to determine cut-offs for ovarian volume and follicle number per ovary for diagnosis of polycystic ovary syndrome in Indian women. J Rep Infer., 19(3): 146-51.

31. Şentürk Ş, Hatirnaz S, Kanat-Pektaş M (2018): Serum Preptin and Amylin Levels with Respect to Body Mass Index in Polycystic Ovary Syndrome Patients. Medical science monitor: Int Med J of Exper and Clin Res., 24, 7517-23.

32. Kayemba K, Armella P, Sidi M (2017): Pelvic MRI as an alternative to pelvic ultrasound for the diagnosis in overweight and obese adolescent girls. Int J Pediatr Adolesc Med., 4: 147-152.

33. Aydoğmuş H, Kelekçi S, Elmalı F et al. (2018): Can we use serum Anti-Mullerian hormone to differentiate the diagnosis between polycystic ovary syndrome patients and healthy women with polycystic ovarian morphology and regular menstrual cycles. Saudi Med J., 39(10), 1011-16.

34. Dewailly D, Lujan M, Carmina E et al. (2014): Definition and significance of polycystic ovarian morphology: a task force report from the Androgen Excess and Polycystic Ovary Syndrome Society. Hum Reprod Update, 20(3), 334-52. 